\title{
HIGH TEMPERATURE NANOINDENTATION OF NI-BASE SUPERALLOYS
}

\author{
A. Sawant ${ }^{1}, \mathrm{~S} . \mathrm{Tin}^{1}$ and J.-C. Zhao ${ }^{2}$ \\ ${ }^{1}$ Illinois Institute of Technology, 10 W. 32 ${ }^{\text {nd }}$ St. Chicago IL 60616 \\ ${ }^{2}$ The Ohio State University, 2041 College Rd., Columbus, OH, 43210
}

Keywords: high temperature indentation, superalloy, single crystal, diffusion multiple

\begin{abstract}
Novel techniques for characterizing and assessing the properties of Ni-base superalloys are becoming increasingly important to the accelerated development of new structural alloys and the advancement of physics-based mechanical property models. Instrumented indentation techniques have long served as a useful approach for probing the mechanical response of a wide range of engineering materials. Nano and micro-indentation techniques are particularly well suited for measuring the properties of materials and structures in which the size or volume of the sample would make conventional mechanical testing methods cumbersome or cost prohibitive. Deformation volumes can be carefully controlled such that indentation can be applied to probe the properties of specific phases or features present within the microstructure. Moreover, recent developments in micromachining of micropedestal specimens via focused ion milling, chemical etching or femtosecond laser ablation allow for the direct assessment of compressive stress-strain responses using indentation equipment. Since high temperature structural materials, such as Ni-base superalloys, are primarily used in a variety of critical applications for turbine engines, materials development and usage of life prediction models has traditionally been extremely conservative. Predictive physics-based deformation models may increase the confidence of the property values and minimize some of the design conservatism, thus allowing more efficient materials usage and contributing to more efficient engine operation. Such predictive models may also accelerate the development of high temperature structural materials with improved temperature capability to further enhance engine performance. Recent developments have led to the ability to conduct instrumented micro/nano-indentation at elevated temperatures. Utilization of high temperature indentation techniques may potentially address some of these challenges associated with understanding the fundamental deformation mechanisms across limited length scales as well as establish new methods of utilizing combinatorial techniques for the design of structural materials.
\end{abstract}

\section{Introduction}

Instrumented indentation has become an important tool that can be used to evaluate the mechanical properties of a wide range of engineering materials across the nano-, micro- and meso- length scales $^{[1-4]}$. The simplicity of the indentation process offers a number of advantages when compared to other experimental approaches for assessing the micro-mechanical properties of materials and structures that possess limited volumes. By varying the indenter tip geometry and applied load, indentation techniques can be used to probe different volumes of material ${ }^{[5-8]}$. Interpretation of the resulting load-displacement curves, however, is non-trivial as the strain fields underneath the indenter tip are extremely complex and sensitive to material processing parameters and microstructure. For meaningful analyses of indentation data, knowledge of both the contact mechanics and underlying structure of the material is required to minimize the occurrence of experimental artifacts that may distort the resulting data. To complement indentation studies, computational modeling efforts are commonly employed to simulate the evolution of the plastic zone beneath the indenter and determine the effective stresses present within the material. Depending on the nature of the problem that is being addressed, different types of models can be applied to simulate the characteristic response of the material during indentation ${ }^{[9-15]}$. For example, atomistic, molecular dynamics (MD) and dislocation dynamics models are often used to quantify the initial events corresponding to plastic deformation and develop theories that elucidate the observed size effects during indentation. Continuum-based models, on the other hand, are often applied to develop qualitative engineering correlations between indentation properties and mechanical properties in bulk materials. Combining these models will serve to assist in bridging the gap between continuum and dislocation plasticity.

For low load micro- and nano- indentation, the area of contact between the indenter and material varies during testing and is indirectly measured using the depth of penetration. Unlike conventional hardness tests, the residual impression left on the surface of the material does not need to be measured as the uniform geometry of the indenter tip combined with precise displacement sensing equipment with nanometer resolution is used to calculate the contact area during indentation as a function of load. The resulting load-displacement curves are then used to quantify the hardness of the material, $\mathrm{H}_{\mathrm{IT}}$, which is defined as the ratio of the maximum load, $\mathrm{P}_{\mathrm{MAX}}$, over the projected area, $\mathrm{A}_{\mathrm{P}}$. In addition to measuring the hardness, indentation techniques are also commonly used to determine the elastic modulus, fracture toughness, flow stress and strain hardening exponent of the material being tested. During indentation, the initial contact between the indenter and sample surface can be considered elastic. As the load increases, plastic flow and yielding occurs beneath the material in contact with the indenter. Once the maximum load is attained, the slope of the unloading curve measured from the tangent of $\mathrm{P}_{\mathrm{MAX}}$ can be analyzed to calculate the elastic modulus of the sample material. For viscoelastic materials, these analyses do not necessarily hold true as the linear relationship between load and penetration depth during unloading may be influenced by parameters, such as the loading/unloading rate as well as the magnitude of the maximum load. A distinguishing characteristic corresponding to viscoelastic materials is the tendency to continue to deform at a constant applied load. The resulting depth change as a function of time is often referred to as indentation creep, although the source of this displacement in certain instances may not be directly attributed to plastic flow. Growth of cracks originating from the indent may also serve to change the displacement of the indenter as a constant 
load. In these instances, the growth of the cracks and fracture toughness of the material can be assessed from the steady state displacement rate when held at $\mathrm{P}_{\mathrm{MAX}}$.

Instrumented indentation techniques are of great interest to scientists and engineers as it enables them to acquire mechanical property measurements from diverse classes of materials. The characteristic properties of biological materials and structures ${ }^{[16}$ ${ }^{17]}$, organic polymers ${ }^{[18,19]}$, metals ${ }^{[20,21]}$, $\operatorname{ceramics}^{[22,23]}$ and electronic materials ${ }^{[24-26]}$ can all be evaluated via indentation. Due to the ease of sample preparation and limited volume of deformed material, indentation tests are often considered to be nondestructive in nature. In addition to yielding direct information pertaining to the mechanical properties of the material, the characteristic shape of the resulting load-displacement curves can also be used to identify non-linear events occurring during indentation, such as pressure-induced phase transformations, coating or film delamination and defect nucleation. One of the fundamental limitations of many instrumented indentation systems is the lack of environmental control. The highly sensitive depth sensing systems that are utilized on most indentation systems requires a stable environment and temperature fluctuations of even a few degrees can influence both the compliance of the system and resolution of the control electronics. Since the systems are designed and calibrated specifically for operation at ambient conditions, the fidelity of the results acquired under non-ambient are typically unreliable. With many engineering structures and devices being utilized at non-ambient temperatures and conditions, the applicability of indentation techniques for these particular materials is limited due to the lack of temperature dependent mechanical properties.

The present investigation has focused on evaluating the high temperature mechanical properties of Ni-base superalloys at elevated temperatures using these instrumented indentation techniques. The anisotropy of a commercial single crystal Nibase superalloy was assessed and used to derive a phenomenological model capable of accounting for the visoelastic effects present at elevated temperatures. Once the methodology for assessing and analyzing the high temperature load - displacement indentation curves was established, the technique was applied to evaluate the mechanical properties of a multicomponent diffusion couple. The interdiffusion zones of the diffusion multiple were probed to correlate variations in hardness and modulus with the graded composition.

\section{Experimental Materials and Procedure}

A MicroMaterials indentation platform shown in Figure 1 was used to conduct high temperature indentation studies. The platform is contained within an environmental chamber purged with high purity $\mathrm{Ar}$ such that $\mathrm{O}_{2}$ levels are maintained below $0.01 \%$ to minimize oxidation of both the sample surface as well as the indenter tip. Square specimens with edge lengths measuring $\sim 10 \mathrm{~mm}$ and a thickness of $3 \mathrm{~mm}$ were mounted onto a refractory ceramic hot block. Resistance heaters located directly beneath the surface of the ceramic hot block were used to provide specimen heating. To minimize transient thermal fluctuations and heat transfer between the specimen surface and indenter tip during testing, a separate resistance heater and thermal control system was used to provide heating of the indenter. The temperatures of the specimen surface and indenter were controlled to within $\sim 0.1^{\circ} \mathrm{C}$. Since both the electronics and overall frame compliance are highly sensitive to minor changes in temperature, a liquidcooled heat shield was inserted to isolate and contain the radiant heat emanating from the hot stage and indenter. The temperature of the MicroMaterials platform and ambient temperature of the environmental cabinet was maintained at $30^{\circ} \mathrm{C} \pm 0.1^{\circ} \mathrm{C}$ during testing. Sharp diamond and sapphire Berkovich indenters were used to assess the hardness and modulus of the superalloy samples as a function of temperature up to $600^{\circ} \mathrm{C}$. A heated diamond Berkovich indenter was used for the majority of tests conducted at temperatures below $500^{\circ} \mathrm{C}$. Due to concerns with oxidation of the diamond tip at temperatures above $500^{\circ} \mathrm{C}$, a heated sapphire indenter was used for all tests conducted above this temperature.

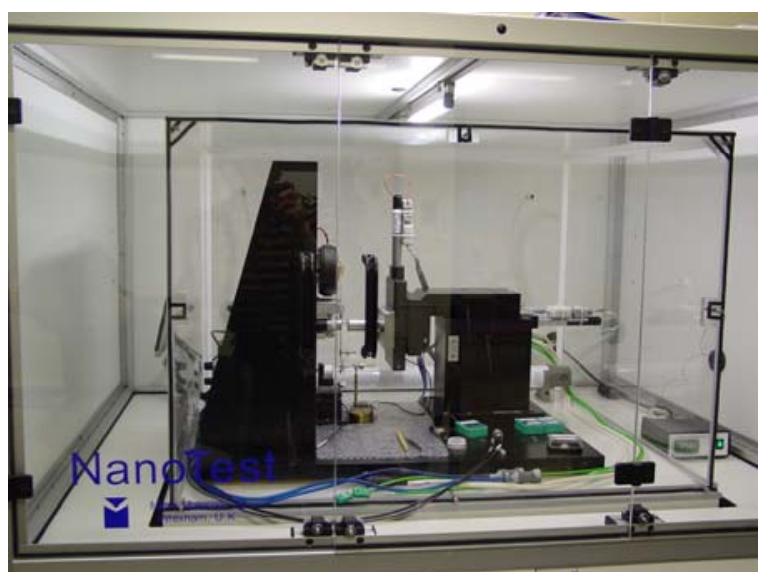

Figure 1: MicroMaterials NanoTest system contained within an environmental enclosure.

Indentation was performed on solution treated and aged single crystal CMSX-4 samples oriented along the $<100>$ and $<110>$ directions as well as a combinatorial diffusion multiple consisting of polycrystalline Ni-based superalloys: IN100, IN718, Rene 95 and ME3. Compositions of the alloys are listed in Table I. The diffusion multiple measured $25 \mathrm{~mm}$ in length and consisted of a $25 \mathrm{~mm}$ diameter cylinder with a $14 \mathrm{x} 14 \mathrm{~mm}$ square opening into which four rectangular $7 \mathrm{~mm}$ X $7 \mathrm{~mm}$ X $25 \mathrm{~mm}$ bars of the constituent alloys were inserted. All of the superalloy samples were sectioned using electro-discharge machining (EDM). The assembly was subjected to hot-isostatic pressing (HIP) cycle at $140 \mathrm{MPa}, 1150^{\circ} \mathrm{C}$ for $1000 \mathrm{~h}$ followed by a water quench. Additional details of this process along with the assessment of the compositional gradients within the interdiffusion zone can be found in Ref [27]. Assembly of the combinatorial diffusion multiple yielded four pairs of diffusion couples: Rene 95 - IN718, Rene 95 - ME3, IN718 - IN100, ME3 - IN100. The finished sample was cut into slices approximately $5 \mathrm{~mm}$ thick and prepared using standard metallographic techniques. To investigate the role of microstructure on the indentation response, the diffusion multiple was evaluated in the as quenched condition, as well after an $1100^{\circ} \mathrm{C}$ exposure for eight hours followed by a furnace cool. The subsequent heat treatment and slow cool allowed the intragranular $\gamma$ ' precipitates within the microstructure of the alloys to coarsen to sizes that are more representative to those found in commercially processed structures. A solution of $33 \% \mathrm{HCl}$, $33 \% \mathrm{HNO}_{3}$ and $33 \% \mathrm{H}_{2} \mathrm{O}$ was used to lightly etch the microstructure of the diffusion multiples and reveal the exact location of the interdiffusion zones.

The load used during indentation was $200 \mathrm{mN}$, while the loading and unloading rates were $3 \mathrm{mN} / \mathrm{s}$ and $9 \mathrm{mN} / \mathrm{s}$ accordingly. The dwell at maximum load varied between 30 and 1500s while 
Table I: Nominal compositions of the alloys (wt.\%) investigated

\begin{tabular}{cccccccccccccccc}
\hline Alloy & Ni & Al & Co & Cr & Fe & Mo & Nb & Ti & V & W & Re & Ta & C & B & Zr \\
\hline Rene95 & 62.4 & 3.5 & 8.0 & 13.0 & - & 3.5 & 3.5 & 3.7 & - & 3.5 & - & - & 0.03 & 0.0015 & 0.03 \\
IN718 & 53.0 & 0.5 & - & 19.0 & 18.5 & 3.5 & 5.1 & 0.9 & - & - & - & - & 0.08 & - & - \\
IN100 & 60.4 & 5.5 & 15.0 & 10.0 & - & 3.0 & - & 4.7 & 1.0 & - & - & - & 0.16 & 0.14 & 0.06 \\
ME3 & 50.9 & 3.4 & 20.6 & 13.0 & - & 3.8 & - & 3.7 & - & 2.1 & - & 2.4 & 0.05 & 0.025 & 0.05 \\
CMSX-4 & 61.2 & 5.6 & 10.0 & 6.5 & - & 0.6 & - & 1.0 & - & 6.0 & 3.0 & 6.0 & - & - & - \\
\hline
\end{tabular}

the dwell to ascertain the thermal drift was set at 40s. Room temperature calibrations to assess system parameters and frame compliance were performed using standard fused silica, steel and elemental tungsten samples. Indentation tests were carried out at $30^{\circ} \mathrm{C}, 200^{\circ} \mathrm{C}, 300^{\circ} \mathrm{C}$ and $400^{\circ} \mathrm{C}$. For the elevated temperature tests, a minimum of six discrete indentation measurements were evaluated at locations corresponding to nominally similar compositions.

\section{$\underline{\text { High Temperature Indentation }}$}

The standard methodology of applying an Oliver-Pharr powerlaw fit to the unloading portion of the load-displacement curve to evaluate the elastic modulus requires that the material being tested exhibit purely elastic behavior; this is a valid assumption for most metallic materials at room temperature.

$$
E_{r}=\left[\frac{1-v_{s}^{2}}{E_{s}}+\frac{1-v_{i n}^{2}}{E_{i n}}\right]^{-1}
$$

Where $E_{r}$ is the reduced or nanoindentation modulus, $E_{s}$ is the modulus of the substrate, $E_{\text {in }}$ is the modulus of the indenter, $v_{s}$ and $v_{\text {in }}$ are the Poisson's ratio of the substrate and indenter, respectively. At elevated temperatures, however, the unloading response associated with many different classes of materials during indentation becomes visco-elastic in nature and the conventional analyses of the load-displacement curves are no longer valid. High temperature nanoindentation studies on materials such as fused silica ${ }^{[5]}$ and bulk metallic glasses ${ }^{[29]}$ have demonstrated the viability the testing technique, but many of the materials investigated exhibit minimal creep rates over the range of temperatures investigated. Polymeric materials, on the other hand, tend to exhibit complex visco-elastic-plastic responses ${ }^{[19]}$ when tested at room temperature and multiple phenomenological approaches ${ }^{[18,28]}$ have been developed to effectively model their behavior and extract useful information from nanoindentation tests on these materials. One of the more common approaches to account for the time dependent deformation in crystalline metallic materials is the utilization of a straightforward two-element Maxwell model ${ }^{[29,30]}$. In the approach, the creep-compliance, $\mathrm{C}_{\text {creep }}$, of the specimen is used to derive an equivalent expression for the change in contact stiffness measured during nanoindentation.

$$
C_{\text {apparent }}=C_{\text {elastic }}+C_{\text {creep }}
$$

where $\mathrm{C}_{\text {elastic }}$ is the elastic compliance of the instrument and $\mathrm{C}_{\text {apparent }}$ is the measured compliance during nanoindentation testing. At room temperature, when the unloading response of most crystalline metals and ceramics can be assumed to be purely elastic, $\mathrm{C}_{\text {elastic }}=\mathrm{C}_{\text {apparent }}$ and $\mathrm{C}_{\text {creep }}=0$. During testing at elevated temperatures, however, $C_{\text {creep }}$ needs to be experimentally measured and incorporated into the analyses to account for the time-dependent deformation inherent within the resulting load-displacement curve ${ }^{[30-32]}$. A direct means of quantifying the creep compliance for a particular temperature, peak load $\left(\mathrm{P}_{\max }\right)$, and loading rate is to incorporate a constant load dwell during the nanoindentation test at $\mathrm{P}_{\max }$. . The resulting depth change of the indenter as a function of time, $d h / d t$, can then be used to calculate $\mathrm{C}_{\text {creep. }}$ The creep compliance, $d h / d P$, can be evaluated as follows:

$$
\mathrm{C}_{\text {Creep }}=\frac{d h}{d P}=\frac{d h}{d t} \cdot \frac{1}{\frac{d P}{d t}}=\frac{\dot{h}_{\text {creep }}}{\dot{P}_{\text {creep }}}
$$

$\mathrm{C}_{\text {creep }}$ can be effectively described as the rate of change of depth per rate of change of load. Since there is no change in load in the case of constant dwell at $\mathrm{P}_{\max }$, the rate of change of load per time can be assumed to be proportional to the loading or unloading rate, $d P / d t$. By applying this compliance correction, the elastic modulus of crystalline metallic materials can be accurately assessed as a function of temperature via nanoindentation.

\section{Results and Discussion}

Depending on the test temperature, dwell time and maximum load, the residual indents from the Berkovich tip appeared as an equilateral triangle with edge lengths measuring between $5 \mu \mathrm{m}$ and $10 \mu \mathrm{m}$. The underlying microstructure of the solution heat treated and aged single crystal CMSX-4 contained a relatively uniform dispersion of cuboidal $\gamma$ ' precipitates possessing edge lengths on the order of $500 \mathrm{~nm}$, while the microstructure of the sub-solvus processed polycrystalline alloys used to form the diffusion multiple typically contained a tri-modal distribution of $\gamma$ ' precipitates. For the single crystal CMSX-4 sample, the comparatively homogeneous microstructure enabled sufficiently large volumes of both the $\gamma$ and $\gamma^{\prime}$ phases to be deformed during indentation and the resulting modulus measurements are representative of the bulk. For the constituent alloys comprising the diffusion multiple, however, the heterogeneity of the $\gamma$ ' microstructure coupled with the random grain orientation prohibited direct correlation of indentation hardness and modulus with the bulk alloy properties at low loads $(<500 \mathrm{mN})$. Higher loads and correspondingly larger deformation volumes were required to accurately assess the bulk properties of heterogeneous microstructures via instrumented indentation.

Figure 2 shows a series of load-displacement curves corresponding to the indentation of $<001>$ oriented CMSX-4 as a function of temperature from $30^{\circ} \mathrm{C}$ to $400^{\circ} \mathrm{C}$. For each of the tests, a dwell of 1500s at $P_{\max }=200 \mathrm{mN}$ was used to assess the depth change or creep compliance as a function of time, $\dot{h}_{\text {creep }}$ 
or $d h / d t$. As expected, the magnitude of the creep displacement during the dwell period was observed to increase as a function of temperature. No significant changes in depth were measured at $30^{\circ} \mathrm{C}$ irrespective of dwell times. At elevated temperatures, the overall creep displacement rate was measured to be relatively constant during the entire duration of the dwell and little evidence of accelerated primary creep strain rates could be detected from the creep displacement curves. The unloading portion of the load-displacement curves for all of the tests appear linear except at low loads where the discontinuity in the slope of the curve can be attributed to thermal drift. After the indentation load - displacement curves were obtained, the Oliver-Pharr analysis was applied to calculate the corresponding hardness and elastic modulus. The load, $\mathrm{P}$, can be expressed in terms of the change in displacement, $h$, using:

$$
P=A^{\prime}\left(h-h_{f}\right)^{m}
$$

where $A^{\prime}$ is a material constant, $h_{f}$ is the depth of the residual impression and $\mathrm{m}$ is an exponent that is to be determined. The true contact plastic depth, $\mathrm{h}_{\mathrm{c}}$, can be expressed as:

$$
h_{c}=h_{\max }-\varepsilon\left(C_{\text {elastic }} P_{\max }\right)
$$

where $\varepsilon$ is the form factor for the indenter. In the case of a Berkovich indenter $\varepsilon=0.75$. Since the contact compliance can be expressed as a function of the reduced modulus and the projected contact area, A:

$$
C_{\text {elastic }}=\sqrt{\frac{\pi}{A}} \frac{1}{2 E_{r}}
$$

Due to the characteristically small indents associated with nanoindentation, diamond area functions (DAFs) are commonly used to relate the depth of the indentation and the projected area of indentation for different indenter geometries. At elevated temperatures, the creep compliance must be accounted for and $\mathrm{C}_{\text {elastic }}-\mathrm{C}_{\text {creep }}=\mathrm{C}_{\text {apparent }}$. Combining equations [1], [3] and [6], the apparent compliance measured during elevated temperature nanoindentation testing be expressed as:

$$
\mathrm{C}_{\text {apparent }}=\sqrt{\frac{\pi}{A}} \frac{1}{2}\left[\frac{1-v_{s}^{2}}{E_{s}}+\frac{1-v_{\text {in }}^{2}}{E_{\text {in }}}\right]-\frac{\dot{h}_{\text {creep }}}{\dot{P}_{\text {creep }}}
$$

Thus by measuring $\mathrm{C}_{\text {apparent}}$, $\dot{h}_{\text {creep }}$, A and combining these values with the known properties of the indenter, we can estimate the modulus of the material being tested, $E_{s}$. In this investigation, it was assumed that the modulus, $E_{\text {in }}$, and Poisson's ratio, $v_{\text {in }}$, for the diamond indenter, remained constant over the range of temperatures reported. Figure 3 shows the load-displacement curve for $<001>$ oriented CMSX-4 tested at $400^{\circ} \mathrm{C}$. Determination of the modulus associated with the uncorrected unloading portion of the curve without accounting for the change in compliance due to creep yields $\mathrm{E}_{400^{\circ} \mathrm{C} \text {, uncorrected }}$ $=140 \mathrm{GPa}$. This is significantly higher than the modulus of the same sample measured at room temperature where $\mathrm{E}_{20^{\circ} \mathrm{C}}=$ 126GPa. Since the modulus of CMSX-4 decreases as a function of temperature, the change in the initial slope of the unloading curve can be attributed directly to creep effects that increase the extent of plasticity occurring during indentation. By incorporating the creep compliance and using Equation [7], the resulting modulus was measured to be 113GPa, which is in close agreement with values reported in literature for CMSX-4 at $400^{\circ} \mathrm{C}^{[14,17]}$. The indentation modulus measurements for single crystal CMSX-4 specimens oriented in the $<001>$ and $<110>$ direction were measured as a function of temperature are presented in Figure 4 and Table II. The measurements are consistent with values reported for bulk specimens of CMSX-4 and clearly distinguish the dependence of the modulus on both orientation and temperature of the single crystal Ni-base superalloy specimen.

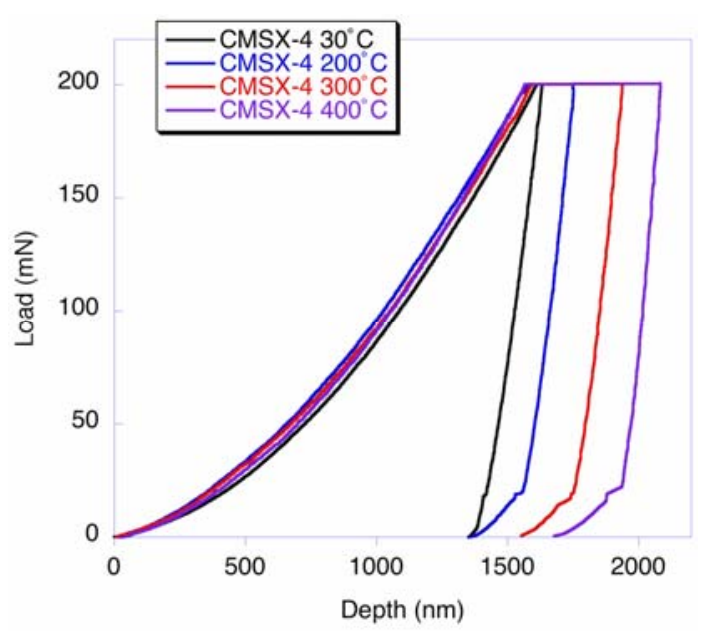

Figure 2: Load displacement curves comparing the indentation response of $<001>$ oriented single crystal CMSX-4 as a function of temperature.

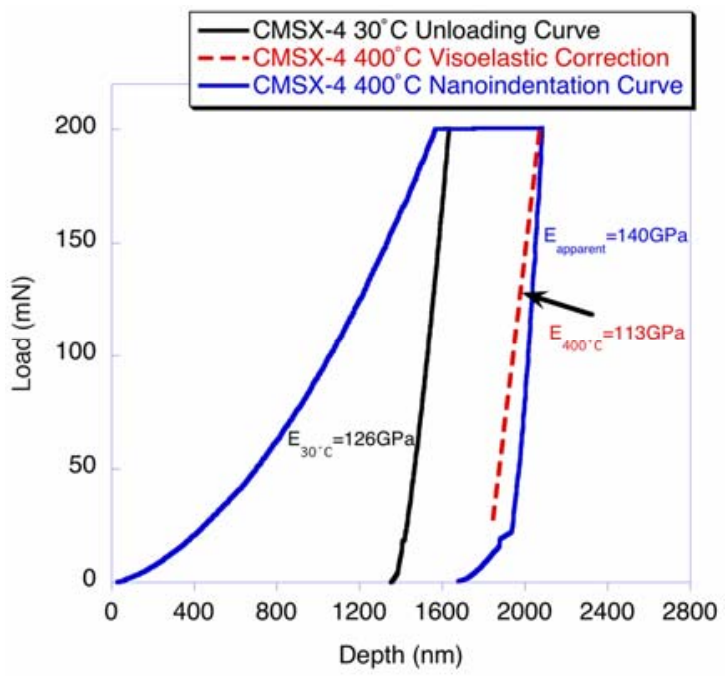

Figure 3: Application of the modified Oliver-Pharr analyses to determine the modulus of $<001>$ oriented CMSX -4 at $400^{\circ} \mathrm{C}$.

Anomalous hardening was observed to occur as a function of temperature with the nanoindentation hardness, $\mathrm{H}_{\mathrm{IT}}$, increasing from $3.83 \pm 0.11 \mathrm{GPa}$ to $4.11 \pm 0.30 \mathrm{GPa}$ when tested at $30^{\circ} \mathrm{C}$ and $400^{\circ} \mathrm{C}$. The increase in hardness a function of temperature can be primarily attributed to the formation of Kear-Wilsdorf locks and macro-kinks within the ordered $\mathrm{L}_{2} \gamma^{\prime}$ precipitate microstructure ${ }^{[19,20]}$. Thermally activated cross-slip of dislocation segments restrict dislocation mobility on the $<110>\{111\}$ slip systems. Continuous hardening of the material occurs as a function of temperature until cube slip along the $<110>\{001\}$ systems become active. 
Indentation measurements were also made across the interdiffusion zones of the combinatorial diffusion multiple. The graded chemical composition present within the interdiffusion zone resulted in corresponding variations in the measured hardness and modulus. The modulus and hardness of the underlying substrate, however, was determined to be highly sensitive to the size, shape and distribution of the $\gamma^{\prime}$ precipitates. Immediately following the HIP cycle, the specimens were rapidly quenched. The high cooling rate was found to induce the precipitation of a fine, uni-modal distribution of intragranular $\gamma^{\prime}$ precipitates, Figure 5a. Since the properties of these polycrystalline Ni-base superalloys are highly dependent upon the underlying size, morphology and distribution of the $\gamma$ ' precipitates, the diffusion multiple specimen was heat treated at $1100^{\circ} \mathrm{C}$ to solution the intragranular $\gamma^{\prime}$ precipitates and allow them to re-precipitate during cooling. The slower cooling rate resulting from the furnace cool was more representative of cooling rates found in large turbine disk rotors and led to the formation of a bi-modal $\gamma^{\prime}$ precipitate distribution, Figure $5 \mathrm{~b}$. Although the measured properties of the diffusion multiple alloys are not directly comparable to commercially processed Ni-base superalloys due to the non-standard heat treatments, these samples can be used to assess the dependence of the alloys on microstructure and localized changes in chemistry. In the present investigation, the modulus and hardness values reported pertain to the diffusion multiple specimens that exhibit a bimodal distribution of $\gamma^{\prime}$ precipitates. Comparison of the hardness and modulus of the interdiffusion zone between IN100 and ME3 at $30^{\circ} \mathrm{C}$ and $400^{\circ} \mathrm{C}$ reveal a slight increase in hardness and a modest decrease in modulus as a function of temperature, Figures 6 and 7. Within the interdiffusion zone, an increase in both hardness and modulus was noted as the indentation measurements traversed over to the side of alloy ME3. This is likely due to the higher overall concentration of refractory alloying additions present in the composition of alloy ME3. Compared to IN100, alloy ME3 contains both additions of W and Ta which impart a high degree of strengthening to the alloy. The characteristic behavior of these alloys is consistent with those of CMSX-4 tested at similar temperatures.

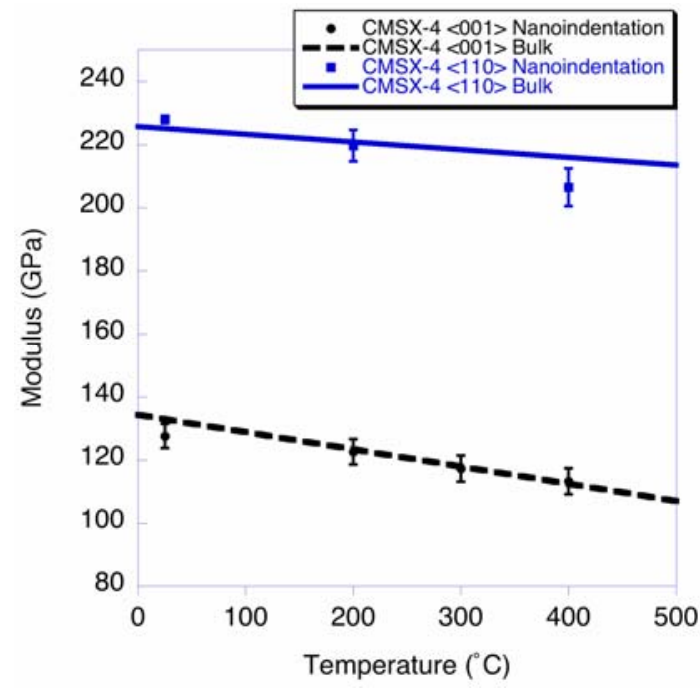

Figure 4: Comparison of the bulk modulus of CMSX-4 and that of CMSX-4 measured via indentation.
The indentation response of the individual constituent alloys comprising the diffusion multiple was also evaluated using the instrumented indentation techniques at temperature between $30^{\circ} \mathrm{C}$ and $400^{\circ} \mathrm{C}$. Although the heat treatments applied to the diffusion multiple were non-ideal when compared to the standard commercial processing route for the individual alloys, assessment of their properties allows for comparative rankings of the alloys. The hardness and modulus of the individual alloys were determined at locations sufficiently distant from the interdiffusion zones of the combinatorial diffusion multiple. Representative load - displacement curves from each of the constituent alloys are shown in Figure 9.

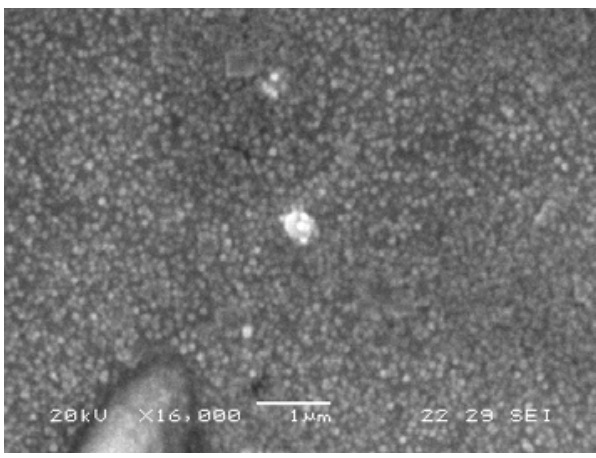

(a)

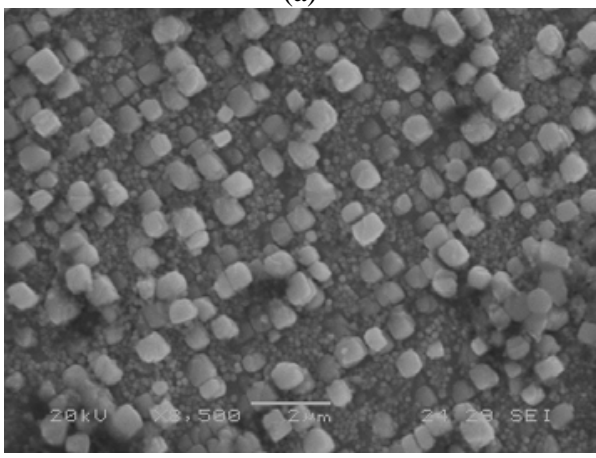

(b)

Figure 5: Scanning electron micrographs showing a (a) unimodal and (b) bi-modal distribution of intragranular $\gamma^{\prime}$ in the interdiffusion zone between IN100 and ME3.

Table II: Nanoindentation modulus measurements for CMSX-4

\begin{tabular}{ccccc}
\hline Sample & $\begin{array}{c}\text { Temperature } \\
\left({ }^{\circ} \mathrm{C}\right)\end{array}$ & $\begin{array}{c}\mathbf{h}_{\max } \\
(\mathbf{n m})\end{array}$ & $\mathbf{P}_{\max }(\mathbf{m N})$ & $\mathbf{E}_{\mathbf{s}}(\mathbf{G P a})$ \\
\hline $\begin{array}{c}\mathrm{CMSX}-4 \\
<001>\end{array}$ & 30 & 1658 & 200.4 & $127.6 \pm 1.2$ \\
$\begin{array}{c}\mathrm{CMSX}-4 \\
<001>\end{array}$ & 200 & 1720 & 196.0 & $122.6 \pm 5.0$ \\
$\begin{array}{c}\mathrm{CMSX}-4 \\
<001>\end{array}$ & 300 & 1950 & 200.0 & $117.3 \pm 6.0$ \\
$\begin{array}{c}\mathrm{CMSX}-4 \\
<001>\end{array}$ & 400 & 2210 & 200.0 & $113.3 \pm 9.0$ \\
$\begin{array}{c}\mathrm{CMSX}-4 \\
<011>\end{array}$ & 30 & 1487 & 200.0 & $227.9 \pm 1.2$ \\
$\begin{array}{c}\mathrm{CMSX}-4 \\
<011>\end{array}$ & 200 & 1335 & 200.3 & $219.7 \pm 5.0$ \\
$\begin{array}{c}\mathrm{CMSX}-4 \\
<011>\end{array}$ & 400 & 1295 & 200.0 & $206.5 \pm 6.0$ \\
\hline
\end{tabular}


Multiple indentation cycles were carried out in close proximity to one another and the values were averaged to determine the bulk properties of IN100, ME3, Rene 95 and IN718. Indentation of these materials under an applied load of $200 \mathrm{mN}$ yielded a symmetric triangular indent with edge lengths that varied between 7 to $10 \mu \mathrm{m}$. Within the interdiffusion zones of the various diffusion multiples, no discrete grains of primary $\gamma$ ' were observed as the microstructure consisted of grains of $\gamma$ with intragranular secondary and tertiary $\gamma^{\prime}$ precipitates. Away from the interdiffusion zones, however, primary $\gamma^{\prime}$ precipitates were observed to be located along the grain boundaries of IN100, ME3 and Rene 95, while $\delta$ phase precipitates were observed within the microstructure of IN718. Considering the volume of the material sampled during indentation along with the heterogeneity of the microstructure, a relatively large amount of intrinsic scatter was noted in the measurements, Table III.

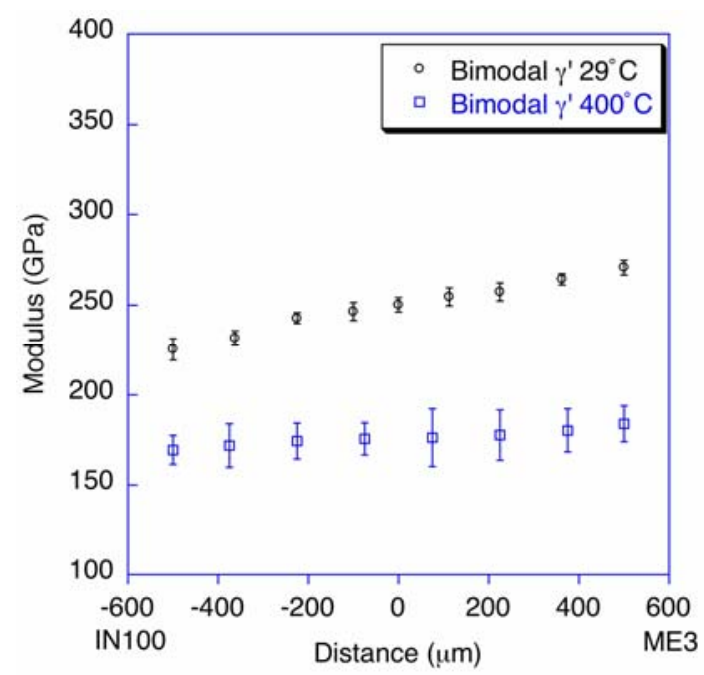

Figure 7: Variation in the modulus measured at $30^{\circ} \mathrm{C}$ and $400^{\circ} \mathrm{C}$ between the interdiffusion zone of IN100 and alloy ME3.

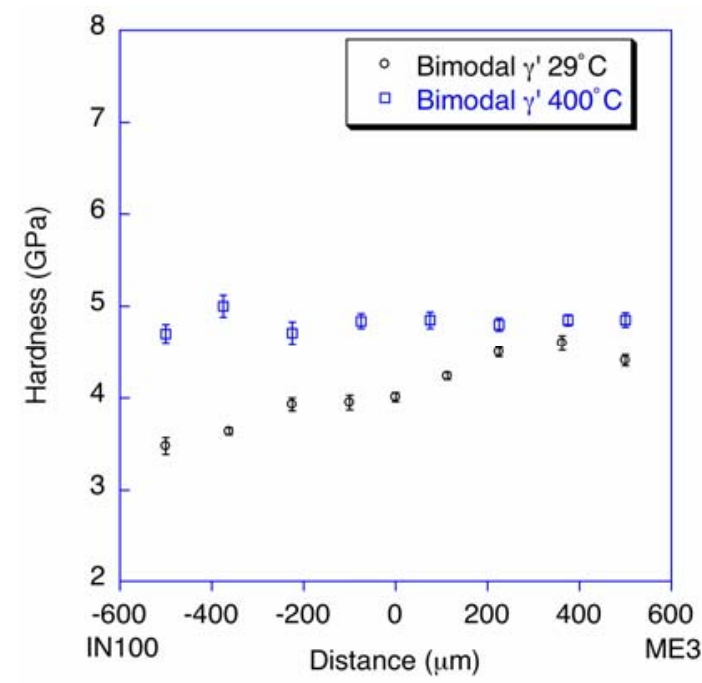

Figure 8: Variation in the hardness measured at $30^{\circ} \mathrm{C}$ and $400^{\circ} \mathrm{C}$ between the interdiffusion zone of IN100 and alloy ME3.

For the tests performed at $400^{\circ} \mathrm{C}$, the modified Oliver-Pharr analysis was used to correct for visco-elastic effects. During the extended dwell at peak load, the creep compliance was measured for the constituent alloys comprising the diffusion multiple, Figure 10. Although the creep compliance may appear

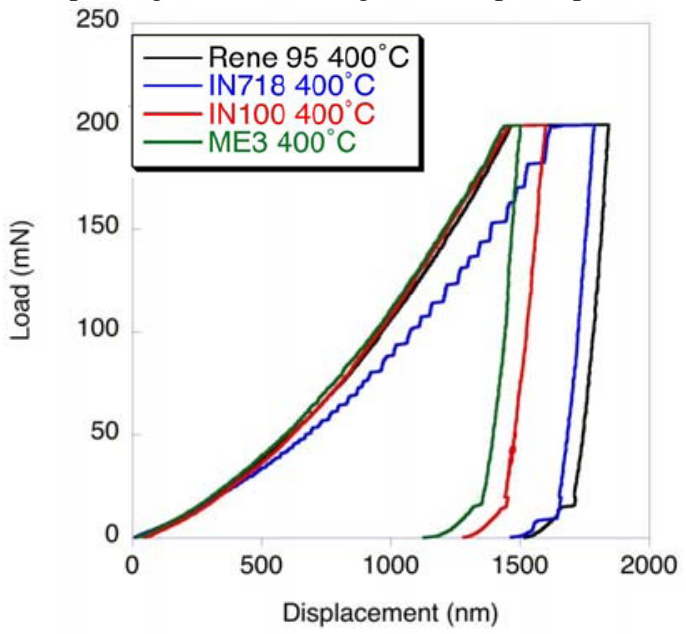

Figure 9: Representative load - displacement curves from the constituent alloys comprising the diffusion multiple.

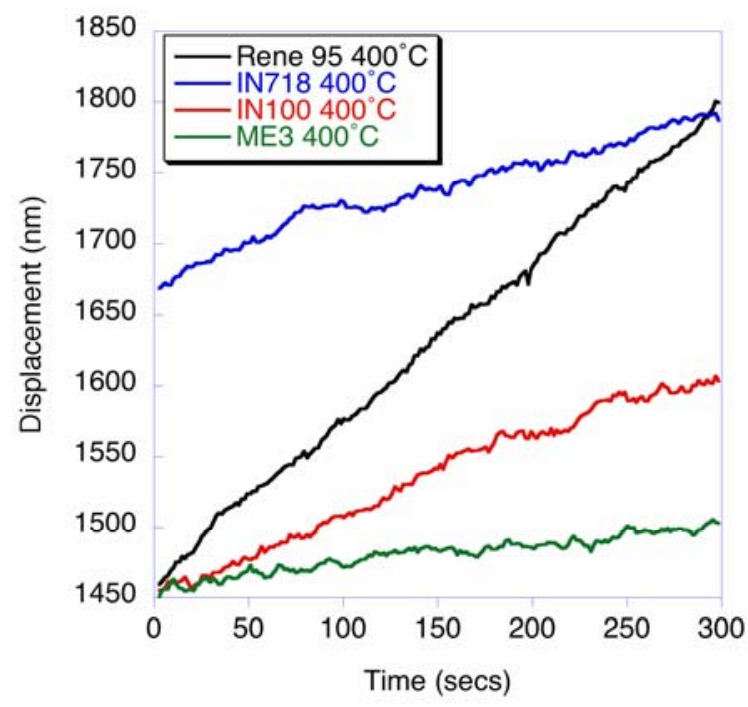

Figure 10: Variation in creep compliance of the constituent alloys comprising the diffusion multiple.

to be a direct measurement of the creep capability of the alloy, these measurements are not directly proportional due to the occurrence of strain gradients during indentation. Moreover, unlike compression of tensile creep tests where the primary stresses are uniaxial, the stresses driving deformation during indentation using sharp Berkovich indenters are multiaxial. Consequently, the creep compliance determined via instrumented indentation cannot be directly compared to the results from uniaxial compression or tension creep properties performed on bulk specimens. The creep performance of the alloys, however, may be assessed qualitatively and ranked against one another. In Figure 10, the creep compliance of alloy ME3 is the lowest suggesting that this alloy is the most creep resistant among group of polycrystalline alloys. Following alloy ME3 are IN100, IN718 and Rene95, respectively, in that 
particular order. Interestingly, the creep compliance of Rene95 was measured to be the highest among these alloys. This is likely due to the irregular thermal processing treatment that was used to modify the microstructure. The temperature of the HIP

Table III: Nanoindentation modulus measurements for constitutent alloys in the diffusion multiple

\begin{tabular}{ccccc}
\hline Sample & $\begin{array}{c}\text { Temperature } \\
\left({ }^{\circ} \mathrm{C}\right)\end{array}$ & $\begin{array}{c}\mathbf{h}_{\max } \\
(\mathbf{n m})\end{array}$ & $\mathbf{P}_{\mathbf{m a x}}(\mathbf{m N})$ & $\mathbf{E}_{\mathbf{s}}(\mathbf{G P a})$ \\
\hline IN100 & 30 & 1566 & 200.2 & $213 \pm 1.2$ \\
IN100 & 400 & 1617 & 200.3 & $161 \pm 9.0$ \\
ME3 & 30 & 1467 & 200.3 & $246 \pm 1.2$ \\
ME3 & 400 & 1797 & 200.3 & $172 \pm 9.0$ \\
IN718 & 30 & 1563 & 200.3 & $228 \pm 1.2$ \\
IN718 & 400 & 1710 & 200.0 & $167 \pm 9.0$ \\
Rene 95 & 30 & 1498 & 200.3 & $219 \pm 1.2$ \\
Rene 95 & 400 & 1505 & 200.0 & $189 \pm 9.0$ \\
\hline
\end{tabular}

cycle, $1150^{\circ} \mathrm{C}$, and the subsequent $1100^{\circ} \mathrm{C}$ exposure did not allow for proper solutioning of the primary $\gamma^{\prime}$ precipitates within the microstructure. As a result, an unusually high percentage of primary $\gamma^{\prime}$ was present in the microstructure resulting in only a small fraction of secondary and tertiary $\gamma^{\prime}$ to form intragranularly. Since the indentation measurements were intentionally used to probe the properties of the microstructure away from the primary $\gamma^{\prime}$ precipitates, the reported creep compliance for this alloy is unusually low relative to the other alloys. Provided that the specimen was heat treated properly to allow for a much higher percentage of $\gamma$ ' to form intragranulary, it is expected that the creep compliance of the alloy would be ranked much higher relative to IN718 and IN100.

Interestingly, the load - displacement curve from indentation of IN718 at $400^{\circ} \mathrm{C}$ was found to exhibit irregular jumps in displacement as a function of load, Figure 11. These "serrations" in the loading portion of the indentation curve correspond to discrete localized yielding events. Similar "serrations" or "pop-in" events have been reported for nanoindentation studies on silicon-based devices or bulk metallic glasses ${ }^{[5]}$. These events have been reported to coincide with the nucleation of mobile dislocations beneath the indenter. These bursts of dislocation activity are also likely to be responsible for the serrated yielding behavior exhibited by IN718 at $400^{\circ} \mathrm{C}$. Interstitial elements within the alloy, such as boron and carbon, are intrinsically mobile within the crystal lattice and are able to rapidly diffuse to low energy locations along the dislocation cores. The application of sufficiently high external stresses allows these dislocations to break free and accelerate a limited distance away from these solute atoms prior to encountering an obstacle or becoming re-pinned by solute atoms. Serrated yielding or dynamic strain aging has been extensively documented to occur is a variety of Ni-base superalloys containing interstitial alloying additions ${ }^{[33,34]}$. The onset of serrated yielding during indentation of IN718 serves as an important indicator corresponding to the onset of plasticity during deformation. One of the major challenges currently associated with nanoindentation measurements is the ability to correlate the measured hardness and modulus to meaningful bulk material properties. Due to the formation of strain gradients under the indent and the presence of multiaxial stresses, robust engineering or physics-based correlations that can be applied to assess flow stresses from indentation hardness values are currently limited. As nucleation of dislocations occurs ahead of the indenter tip during the initial stages of plastic flow, detecting the corresponding depths and respective strain levels associated with the onset of these events is a challenge in most materials. Since the occurrence of serrated yielding events reveal the actual displacement required for initiating dislocation activity, this characteristic yielding behavior may potentially enable the development of accurate models describing the stresses

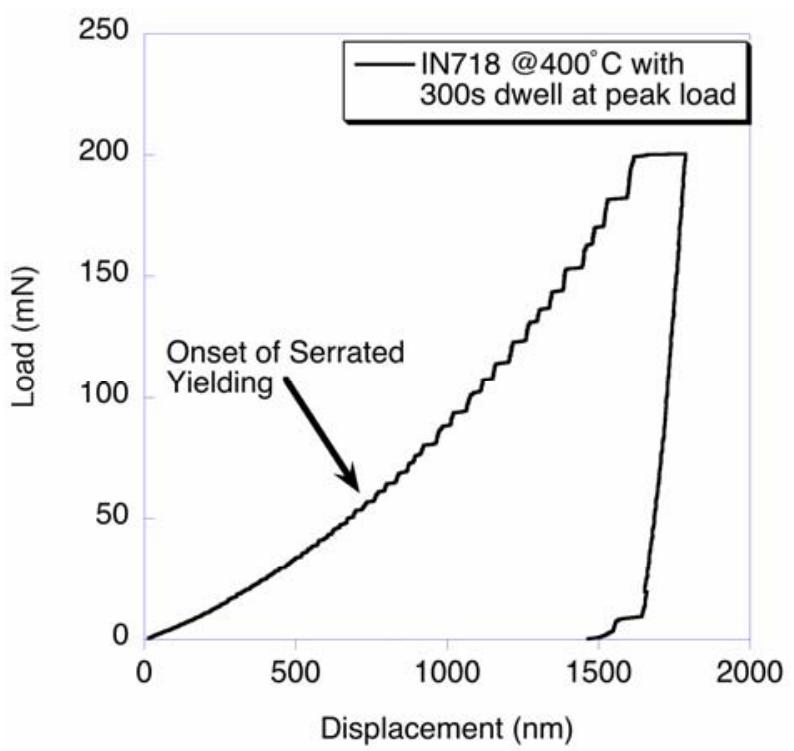

Figure 11: Load - displacement curve corresponding to indentation of IN718 at $400^{\circ} \mathrm{C}$.

\section{Summary and Conclusions}

High temperature nanoindentation is a powerful technique that can be applied to rapidly extract useful mechanical property data from limited volumes of Ni-base superalloys. This unique ability makes this technique ideal for the assessment of small scale site specific mechanical properties in coatings and functionally graded materials. With the appropriate analyses, visco-elastic effects associated with testing at elevated temperature can be addressed and used to determine the modulus and hardness values representative of bulk properties in Ni-base superalloy specimens. However, a number of technical challenges, however, still remain in the development of high temperature indentation techniques as the ability to accurately collect and process meaningful experimental data becomes increasingly sensitive to external test parameters. Preventing metallurgical reactions between the specimen and indenter becomes increasingly difficult as the temperature increases the chemical activities of the respective materials. Moreover, oxidation rates of the specimen are enhanced and control of the testing environment also needs to be addressed. Substantial effort is being dedicated towards resolving many of these issues, as the potential impact of a robust high temperature nanoindentation system may serve to assist in the discovery of new classes of structural materials as well as provide fundamental understanding of how materials accommodate deformation across multiple length scales. 
The application of high temperature nanoindentation techniques with combinatorial diffusion multiples ${ }^{[21]}$ may potentially expedite the discovery of new classes of structural alloys and materials for high temperature service. The interdiffusion zones within these combinatorial diffusion multiples possess compositionally graded microstructures. When combined with localized analysis of chemistries, indentation techniques can be utilized to probe the mechanical properties within the interdiffusion zone and determine how the properties change as a function of temperature. These techniques can also be applied to assess the location specific properties of other functionally graded materials and coating systems. For example, the performance and efficiency of advanced gas turbine engines has become increasingly reliant on the performance of ceramic thermal barrier coatings that reduce the maximum service temperature experienced by the high pressure turbine blade substrate. As the life of coating is dependent on the performance of a variety of interfacial reactions within the layers of the coating system, knowledge of the micro-mechanical properties within these layers will enable the development of life prediction criteria with improved accuracy. Furthermore, high temperature indentation may also be utilized to quantify the creep capability of materials along limited length scales. Variations in the geometry of the indenter coupled with novel specimen geometries can be used to extract a variety of small-scale mechanical properties. In conjunction with complementary multiscale modeling activities, the implementation and interpretation of high temperature nanoindentation is likely to contribute significantly to the scientific understanding of how the mechanical properties and micro-/nanostructure are interrelated.

\section{References}

1. Doerner, M.F. and Nix, W.D., "A method for interpreting the data from depth-sensing indentation instruments", J. Mater. Res., (1986). Vol. 1. p. 601609.

2. Fischer-Cripps, A.C., Nanoindentation. 2nd ed. 2004, New York: Springer-Verlag LLC.

3. Gouldstone, A., Chollacoop, N., Dao, M., Li, J., Minor, A.M., and Shen, Y.-L., "Indentation across size scales and disciplines: Recent developments in experimentation and modeling" Acta. Mater., (2007). Vol. 55. p. 4015-4039.

4. Oliver, W.C. and Pharr, G.M., " An improved technique for determining hardness and elastic modulus using load and displacement sensing indentation experiments", J. Mater. Res., (1992). Vol. 7. p. $1564-1583$.

5. Beake, B.D. and Smith, J.F., "High temperature indentation testing of fused silica and other materials", Phil. Mag. A, (2002). Vol. 82. p. 2179-2186.

6. Schuh, C.A., Lund, A.C., and Nieh, T.G., "New regime of homogeneous flow in the deformation map of metallic glasses: elevated temperature nanoindentation experiments and mechanistic modeling", Acta. Mater., (2004). Vol. 52. p. 58795891.

7. Oyen, M.L., Acta. Mater., " Sensitivity of polymer nanoindentation creep measurements to experimental variables " (2007). Vol. 55. p. 3633-3639.

8. Strojny, A. and Gerberich, G.G., "Experimental analysis of viscoelastic behavior in nanoindentation", Mater. Res. Symp. Proc., (1998). Vol. 522. p. 159164.

9. Oyen, M.L. and Cook, R.F., "Load displacement behavior during sharp indentation of viscous elasticplastic materials", J. Mater. Res., (2003). Vol. 18. p. 139-150.

10. Feng, G. and Ngan, A.H.W., "Effects of creep and thermal drift on modulus measurement using depth sensing indentation", J. Mater. Res., (2002). Vol. 17. p. 660-668.

11. Ngan, A.H.W. and Tang, B., "Viscoelastic effects during unloading in depth sensing indentation" J. Mater. Res., (2002). Vol. 17. p. 2604-2610.

12. Tang, B., Ngan, A.H.W., and Lu, W.W., "Viscoelastic effects during depth-sensing indentation of cortical bone", Phil. Mag., (2006). Vol. 86. p. 5653-5666.

13. Ngan, A.H.W., Wang, H.T., Tang, B., and Sze, K.Y., "Correcting power law viscoelastic effects in elastic modulus measurements using depth sensing indentation", International J. Solids and Structures, (2005). Vol. 42. p. 1831-1846.

14. Takagi, H., Masami, F., and Kakehi, K., " Measuring Young's modulus of Ni-based superalloy single crystals at elevated temperatures through microindentation", Mater. Sci. Eng. A, (2004). Vol. 387-389. p. 348-351.

15. Li, W.B. and Warren, R., "A model for nanoindentation creep" Acta. Metall. Mater., (1993). Vol. 41. p. 3065-3069.

16. Harris, K., Erickson, G.L., Sikkenga, S.L., Bretnall, W.D., Aurrecoechea, J.M., and Kubarych, K.G. in Superalloys 1992. Eds. S.D. Antolovich, R.W. Strusrud, R.A. MacKay, D.L. Anton, T. Khan, R.D. Kissinger, and D.L. Klarstrom, (1992). Warrendale, PA: TMS. p. 297-306.

17. Sieborger, D., Knake, H., and Glatzel, U., "Temperature dependence of the elastic moduli of the nickel-base superalloy CMSX-4 and its isolated phases", Mater. Sci. Eng. A, (2001). Vol. 298. p. 2633.

18. Goodall, R. and Clyne, T.W., "A critical appraisal of the extraction of creep parameters from nanoindentation data obtained at room temperature" Acta. Mater., (2006). Vol. 54. p. 5489-5499. 
19. Pope, D.P. and Ezz, S.S., Int. "Mechanical properties of Ni3Al and nickel-base alloys with high volume fraction of gamma-prime", Met. Rev., (1984). Vol. 29. p. 136-167.

20. Veyssiere, P., "Yield stress anomalies in ordered alloys: a review of microstructural findings and related hypotheses", Mater. Sci. Eng. A, (2001). Vol. 309310. p. 44-48.

21. Zhao, J.-C., "The diffusion-multiple approach to designing alloys", Ann. Rev. Mater. Res., (2005). Vol. 35. p. 51-73.

22. Zeng, $\mathrm{K}$ and Rowcliffe, D., "Analysis of penetration curves produced by sharp indentations on ceramic materials", Philosophical Magazine A, Volume 74, Issue 5 November 1996 , p. 1107 - 1116.

23. Bahr, D.F., Kramer, D.E and Gerberich, W.W., "Nonlinear deformation mechanisms during nanoindentation", Acta Materialia, Volume 46, Issue 10, 12 June 1998, Pages 3605-3617

24. T. Y. Tsui, G. M. Pharr, J. "Substrate effects on nanoindentation mechanical property measurement of soft films on hard substrates", Mater. Res., Vol. 9, 1999, p. $42-46$

25. Smith, G.S., Tadmor, E.B., Bernstein, N., and Kaxiras, E., "Multi-scale simulations of silicon indentation", Acta Materialia, Volume 49, Issue 19, 14 November 2001, Pages 4089-4101

26. Jang, G.W., Lee, J.W. and Duh, J.G., "The nanoindentation characteristics of intermetallics in a solder bump", J. Electronic Mater., Volume 33, Number 10 / October, 2004, p. 1103-1110.

27. Campbell, C.E., Zhao, J.-C., and Henry, M.F., "Comparison of experimental and simulated multicomponent Ni-base superalloy diffusion couples", Journal of Phase Equilibria and Diffusion, Volume 25, No. 1, February, 2004, p. 6-15.

28. Sawant, A. and Tin, S., "High temperature nanoindentation of a Re-bearing single crystal Ni-base superalloy", Scripta Materialia, Volume 58, Issue 4, February 2008, Pages 275-278

29. Pharr, G.M., "Measurement of mechanical properties by ultra-low load indentation", Materials Science and Engineering: A, Volume 253, Number 1, 30 September 1998 , pp. 151-159

30. Chiu, Y.L., Ngan, A.H.W., "Time-dependent characteristics of incipient plasticity in nanoindentation of a $\mathrm{Ni}_{3} \mathrm{Al}$ single crystal", Acta Materialia, Volume 50, Issue 6, 2 April 2002, Pages 1599-1611
31. Liang, H.Y., Woo, C.H., Hanchen, H., Ngan, A.H.W., and Yu, T.X., "Dislocation nucleation in the initial stage during nanoindentation", Philosophical Magazine, Volume 83, Issue 31 - 34 November 2003 , pages 3609 - 3622

32. Fischer-Cripps, A.C., " A simple phenomenological approach to nanoindentation creep", Materials Science and Engineering A, Volume 385, Issues 1-2, 15 November 2004, Pages 74-82

33. Lloyd, D J, Chung, D W and Chatervedi, M. C., "Serrated yielding in a super alloy /40Co-38Ni-17Cr5Ti/ ", Acta Metallurgica. Vol. 23, 1975, pp. 93-100.

34. Rao, K.B., Kalluri, S., Halford, G.R. and McGaw, M.A., "Serrated flow and deformation substructure at room temperature in Inconel 718 superalloy during strain controlled fatigue", Scripta Metallurgica et Materialia, Volume 32, Issue 4, 15 February 1995, Pages 493-498 